\title{
A Digital Twin for Directing People Flow in Preserved Heritage Buildings
}

\author{
Armando Trento $^{1}$, Gabriel Wurzer ${ }^{2}$, Ugo Maria Coraglia ${ }^{3}$ \\ ${ }^{1}$ Sapienza University of Rome ${ }^{2}$ TU Wien ${ }^{3}$ Sapienza University of Rome \\ 1,3\{armando.trento|ugomaria.coraglia\}@uniroma1.it ${ }^{2}$ gabriel.wurzer@tuwien. \\ ac.at
}

This paper showcases a Digital Twin in the form of a simulation interacting with a BIM. As will be shown, such a combined can serve specific problems during building operation (here: directing people flow in preserved heritage buildings) .

\author{
Keywords: People Flow, Heritage Buildings, Agent-Based Simulation, Building \\ Information Model, Digital Twin
}

\section{INTRODUCTION}

There are many buildings which fall under heritage preservation; among these are cultural institutions and museums which are frequented by a high amount of pedestrian traffic which must be directed for optimal access, security and safety reasons, and changing usage scenarios.

Solutions such as belt barriers offer a highly reconfigurable solution, however, they come at a high cost in terms of personnel. A further disadvantage lies in the fact that they do not blend in well, making the access area look like a security check rather than an integral part of the historical building.

This paper argues for retractable crowd control solutions designed to fit into the building's style. Instead of using personnel for reconfiguration of the access area, we entrust the building itself with the responsibility to manage people flow (see figure 1a) through use of an Agent-Based Simulation (ABS) acting on top of the Building Information Model (BIM), thereby forming a Digital Twin.

Our contribution lies in the following areas:

- We initially review the State of the Art in directing flows in heritage buildings (see "Back- ground and problem definition") in order to establish a methodology for sentient buildings that are able to direct pedestrian flow (see "Methodology").

- We describe an ABS acting on top of a BIM, which computes the next state of actuators (e.g. barriers) using current and recorded pedestrian flow as well as preset rules (see "Digital Twin").

- We showcase the approach using the concrete example of the Palazzo della Civiltà Italiana in Rome (see "Case Study"), relating the results back to our Methodology and once again looking at the apparent discrepancy between heritage protection and implementation of solutions for management of people flow (see "Discussion").

As further contribution, this paper shows how a Digital Twin can be used in practical terms - an undertaking which has often been named in literature but hardly ever done to the extent brought forward herein. 

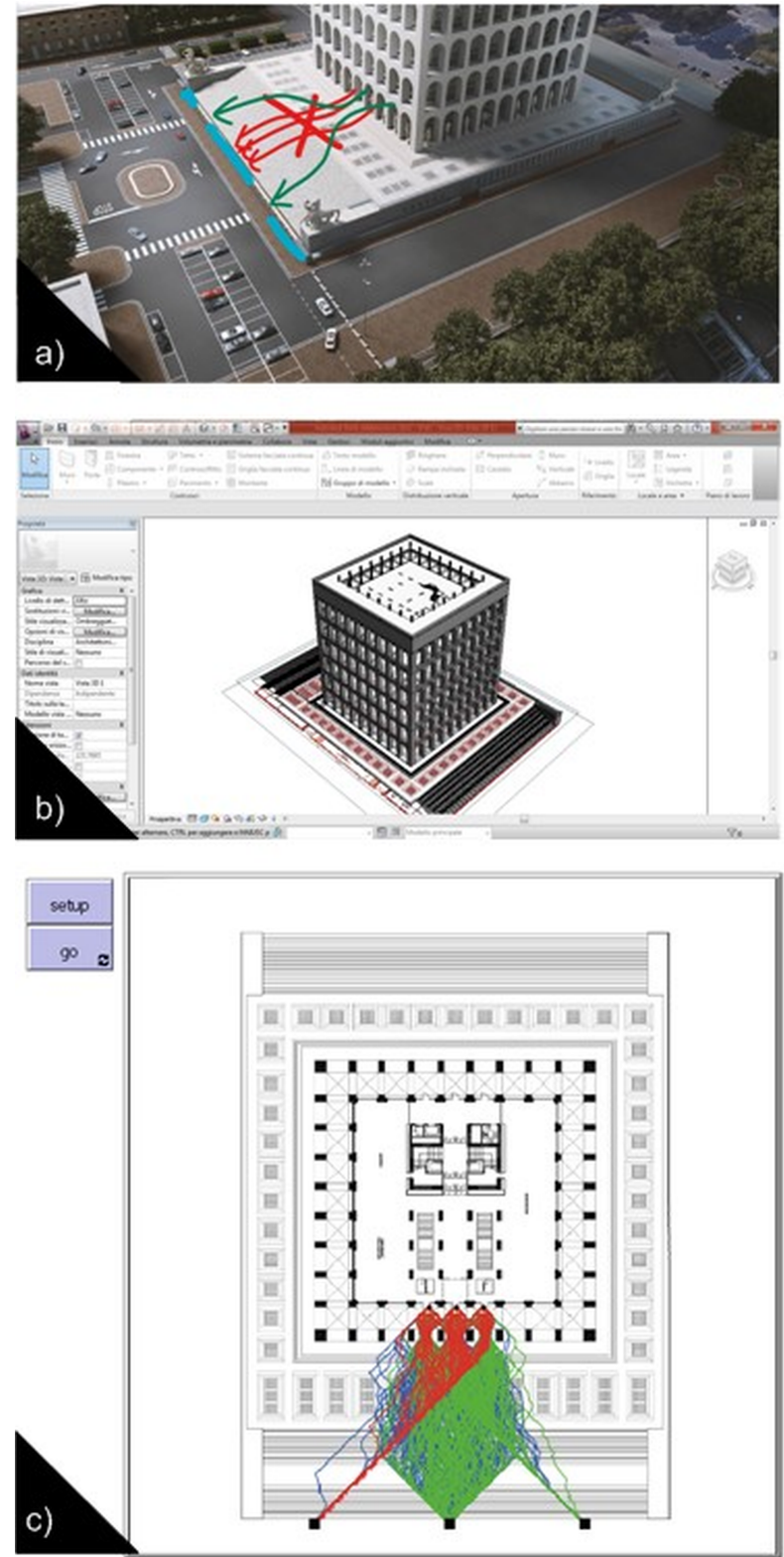

Figure 1

(a) Concept of Digital Twin for directing pedestrian flow to three exits at closing times (b) BIM representation of the building (c) Screenshot of ABS acting on top of BIM, actuating retractable barriers based on exit flow 


\section{BACKGROUND AND PROBLEM DEFINI- TION}

A relatively high number of research work has been analyzed by the authors regarding methods and technologies for supporting design processes by enhancing the interaction between existing architectural components/spaces and their Digital Twin model.

Sharma and Tabak (2008) developed an ABS for design of spaces, both for static and dynamic cases. the model allows time-dependent analysis of flow incorporating dynamic route-choice models, agent behaviours and interaction, as well as stochastic variations. Al-Qattan (2016) developed an interactive and a responsive Tangible User-Interface (TUI) for parametric and Building Information Modeling (BIM) applications. The link between both environments associates physical objects with their digital design information to assist users in the digital design process. Haojie Hu et al. (2017) break down space into components and measure each with the straight sight-line of individuals, using Space Syntax Theory (SS-T) for simulations. They conclude that with the assistance of SS-T, Multi Agent Systems (MAS) can be more functional for solving sophisticated problems in real-life environments.

In the context of heritage buildings, implementation of BIM-based tools/methodologies is quite recent, and just few research results can effectively support designers in addressing users' behavior in the building. Intervention on old buildings, and even more the protected ones, has a dual, apparently opposite, goal: on one side the preservation of the existing values, starting from the testimonial evidence of the physic components; on the other side the renovation of functions, according to radically new activities to be hosted. Protection and restructuring/modification lay on the same direction, with opposite verses: a complex complementarity.

In fact, once recognized the artistic valour of the edifice itself and assumed the intrinsic value regarding the immutability of preserved elements, a consolidated philosophy for restoration requires the in- tervention to be: recognizable and reversible (Brandi 2005 [1963]).

Since the building lifecycle defines always changing requirements, the status of these solutions has to be reconfigured accordingly. Fences, belt barriers, advises, signals, lights constitute a kind of solution frequently implemented in practice, designed to be temporary and removable. Directing flows in a protected building, e.g. in the access area, is a key task for supporting different usage scenarios in efficient and safe conditions.

Simeone et al. (2016) have worked on behavioural simulation for heritage buildings use planning, integrating BIM with Game Engine. In their paper they claim that by distributing Artificial Intelligence among virtual users, process entities and building components they can provide the direct measurement of the impact on the artefact. However, this approach does not take into account the interface with the building, and vice versa, missing the requirement for a non-planned (spontaneous) use.

In the research framework presented in this paper, the authors goal is to grant the building itself with the responsibility to facilitate the management of the users flow in real time, also including local variations from the planned use.

\section{METHODOLOGY}

At the moment, the process of identifying and implementing a defined physical framework related to a specific usage scenario is relying only on:

- designer's implicit (function-action) knowledge;

- health and safety personnel, acting on the basis of the designers' predefined configuration.

This work aims at the definition of methodologies and tools for being able able to work on top of heritage BIMs in order to:

- make building usage knowledge explicit, based on data collection (here: of building user behaviour);

- implement a sensor-actuator system in or- 
der to make the automation system support users' needs.

In that sense, this paper transitions from a purely manual scenario to one augmented/aided by a building automation system, enabling the building spaces/components to sense, reason and act (perform?) a required usage variation.

The general methodology for the technical implementation relies on the development of a system that uses sensors as input, an algorithm as intermediate black-box and messages to actuators as output:

- Sensors are used for recording people flow. Among the options in that context are (a.) explicit sources such as proximity sensors, access control crosses, or implicit sources, such as analyzed footage from visual or thermographic cameras, cash-desks, etc.

- The algorithm is generally a control system which has to reason over the sensory inputs for generating messages to actuators. In this case, we employ ABS as reasoning layer on top of a BIM.

- Actuators are used to influence the people flow physically. The modulation can use physical obstacles such as barriers, but also non-blocking elements such light tiles, signals/signs, dynamically projected content, etc.

\section{DIGITAL TWIN}

A Digital Twin is a model of a building which interfaces to the real building and vice versa. In our case, this is done by having the model of the building in a BIM (figure 1b) on top of which an ABS is used for algorithmic decision making (figure 1c). In more precise terms, our ABS initially loads a schema of the building which is a simplified floor plan. It then connects to input sensors on which it is ready to receive the current flow rate. In our case, these readings are artificially emulated by assuming a certain flow rate which changes through time. In practice, any occupancy sensor (direct - e.g. light barrier, turnstile; indirect - e.g. through an infrared camera whose image is processing via computer vision to get the amount of persons passing a specific [virtual] line) could be used.

After obtaining the current exit flow, the ABS then computes the amount of people which will fit through a retractable barrier comfortably in real time by (1.) predicting speed and arrival at the currently opened barriers which are assigned randomly to each simulated pedestrian, which in turn (2.) gives a density per barrier. If this density surpasses a threshold, then a next barrier is scheduled to open if possible. The decision can still be (3.) overturned by rules such as predefined schedules that define availability of barriers over the course of the day (example: max one barrier at the evenings, no barriers open during the night).

In cases where there are large fluctuations in the observed flow, one can also use historical data for that specific day and time to get an estimate; this usually happens in museums, where the time of payment acts as an estimator for inflow.

After reaching a decision, the ABS translates this to a message to be sent to the actuators (here: retractable barriers). To avoid constant fluctuations, it is usual to employ a hysteresis (implemented e.g. by introducing a voluntarily lag between triggering and executing the command sent to an actuator, which extends the lag or cancels the request if converse commands are received in the time window).

The opposite case, namely that a physical override (e.g. button which sets the state of a barrier) dictates the state of the Digital Twin, is also possible. For this to be possible, such manual switches must be interfaced to the ABS as well.

As side-note, a simulation model acting on the real world without validation is just an assumption; it would be useful to validate the computed assumptions by comparison with sensory data - here: The predicted exit flow with the actual exit flow - in order to establishing a feedback loop. However, since our case study was conducted purely in silico, it would not have made sense to reproduce our assumption (i.e. self-fulfilling prophecy). This aspect is thus left 
for the physical implementation which the author of this paper aim to do undertake in the near future.

\section{CASE STUDY}

Palazzo della Civiltà Italiana in Rome is a relevant historical building also known as "Squared Colosseum". This public property iconic building of particular historical interest, protected by Minister of Cultural Heritage, initiated with a design competition in 1935 for the planned 1942 world exhibition.

The Palazzo was designed by the architects $G$. Guerrini, E. B. La Padula and M. Romano and constructed between 1938 and 1943. It was inaugurated on November 1940 as the centrepiece of the Esposizione Universale di Roma neighbourhood (EU42). The structure is also considered one of the most representative examples, in the modern history of architecture, of rational synthesis of classical linguistic elements.

The Squared Colosseum consists of a box that stands on a wide base connected to the street level by two opposing staircases. The scale is imposing: the base covers an area of 8,400 square meters, and the building has volume 205,000 cubic meters with a height 68 meters ( 50 meters from the base; each level is 8 meters high).

The four faces of the prismatic solid, covered with slabs of travertine stone, are characterized by the strong rhythm of the arches in sequence (nine repeated openings for six floors), strong architectural motif that makes it instantly recognizable as one of the symbolic monuments of Rome.

Recently, FENDI, an international company known in the fashion sector, moved there its offices headquarter, renting the full building, including six floors used as open-space offices and a ground floor used as internal Cafè. The agreement with the Municipality reserves the first floor, on top of the stairs podium, to the public use, mainly for exhibitions free of charge.

Every day, because of its mixed use, the building hosts up to one thousand of persons, simultaneously: around 500 employees in the offices, plus 100 exter- nal consultant, and up to 400 visitors of the exhibition.

The problem with controlling the people flow from and to that building is becoming a relevant one. Specifically, the internal flows are comfortably defined by the ample spaces, large stairs and optimised management system for the four lift, but the access from the street to the external monumental stairs and from these to the internal reception area, can be critic under certain conditions.

For instance, it can happen that - non necessarily in case of an extraordinary risky event - a relevant charge of Palazzo inhabitants should get out at the same time, while the external barriers, standing in front of the first ground level step, are closed according to local regulation.

In order to satisfy requirement for both, health and safety and security control, the flow must pass by the main three doors, glide by the external stairs and converge toward the adequate passages on the external barrier.

Controlling that everybody is out, and nobody gets in without permission, in the context of this wide space, when the night comes and when a crowd of pedestrian are moving around is a task that must be solved by enabling the building itself to automatically optimise - in real time - the appropriate number of passages.

As for technical solutions, there is currently a BIM model which was produced in course of a project (Trento et al. 2014), however this is not being used to its full extent in practice; in this paper, we have therefore chosen to base our solution to the problems mentioned before using that BIM model as a basis, so as to point out its potential also in building automation and control. The conducted case study is thus aimed at both at a scientific audience as well as building owners/operators (i.e. the E.U.R. district administration which is part of the city of Rome).

Since getting permission for setting up sensors and actuators is a lengthy process even for a test case - not only because of heritage preservation but e.g. also due to privacy reasons connected to the EU Gen- 
eral Data Protection Regulation (GDPR) - we opted to emulate these parts in silico as part of the ABS.

Out of the existing BIM model we exported a floor plan of the ground as bitmap, rasterized to $0,5 \mathrm{~m} / \mathrm{pixel}$. Considering that the shoulder length of a pedestrian is roughly in that range makes this approach feasible for use in a people flow simulation. The floor plan (see again figure 1c) is roughly divided into (1.) the access area, where we have three retractable barriers, (2.) the three entrances to the building and (3.) the inner area of the building itself.

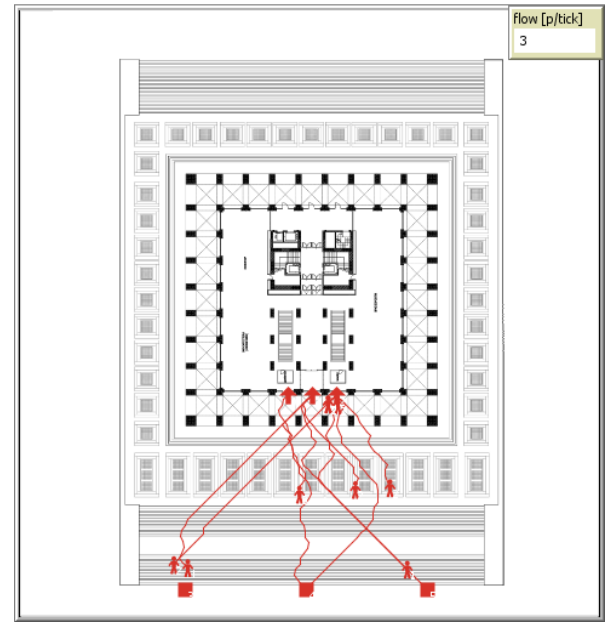

The principal operation of the simulation is to generate agents at a certain (customizable) rate in the building entrances who want to get to the open barriers (figure 2). This rate is read either dynamically from an external source (i.e. real-time input or recorded input) or manually within the simulation. To direct agents we used static routing along the shortest path without computation of congestions and blockages due to physical contact among and between pedestrians and their environment. This approach is reasonable for cases not involving emergencies, however, for such high-density situations proper pedestrian dynamics algorithms (e.g. Helbing et al. 2000) would be needed.
The "sensors" and "actuators" are modeled as own type of agent in our simulation:

- Sensors measure the average rate in a configurable time window (here: 10 "ticks" of the simulation) and along a virtual line that spans all three entrances acting as "start";

- Actuators are agents modeling retractable barriers; they can be in either "open" or "closed" state and can receive requests for setting their state.

The core of the simulation then lies in predicting the density in a configurable time window by examining the computed paths for all agents and estimating their time of arrival. If a configurable density within the time window is surpassed, then a request for opening another barrier is issued. The request is processed by a second stage which assumes it is either day (therefore letting the request pass since the building is principally open), closing or night time (denying the request). A physical override is modelled by three tristate switches (true/false or not set) in the simulation, which gives the final processing stage that always wins. Once a decision has been made it is sent to all actuators that need to be changed. The request is carried out immediately (no hysteresis). As a result, we can see roughly three "epochs" of pedestrian flow (refer again to figure 1c): One where there is only one barrier open, one with two and lastly one with three.

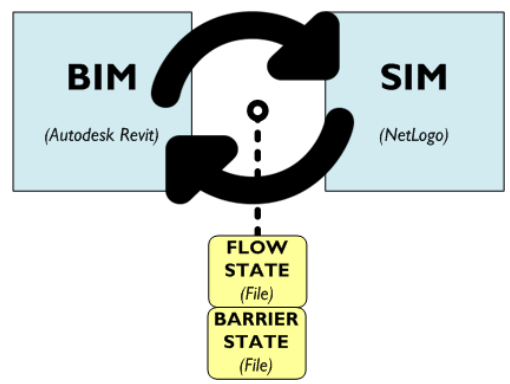

Figure 2

Simulation

Figure 3

System Diagram 
Figure 4

The export of state from the BIM happens through a custom algorithm in Dynamo

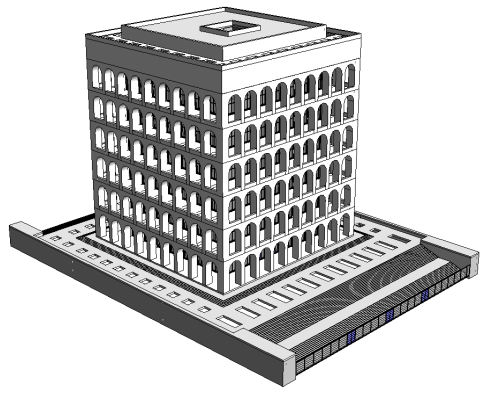

\section{DISCUSSION}

Seeing a Digital Twin as a black-box between sensors for input and actuators for output, as this work has done, is not always the meaning in which authors refer to it. The main intention is a replication of a real system and a representation (i.e. model), sometimes with (uni- or bi-directional) interfaces between the two. Technically, this is accomplished by two shared files (one for the flow, one for the barrier state, see figure 3) to which both BIM and simulation can write. The BIM side is handled by Dynamo in Revit (figure 4), the simulation side by NetLogo (figures 1c and 2). The files can also be updated from an external source ("sensors") and thus allows for real-time interfacing, which is currently being emulated in NetLogo for testing purposes.

Especially in heritage, the choice of sensors and actuators is limited: Issues of heritage protection, reversibility of interventions, privacy protection, ruggedness for use in outdoor environments or simply staffing problems come into play. On the other hand, the Digital Twin proposed herein offers a possibility for managing people flow for sake of safety and security, different space utilization scenarios and proper night-time closing procedures. This now stands in contradiction to the all points mentioned before. However, both points should not be viewed as contradictory, there needs to be a synthesis which can only happen between building professionals running the Digital Twin and the build- ing owner/operator, regulation-makers and, last but not least, the wider public using the building. A closed-loop (fully autonomous) system will certainly be problematic in that case, which is why we have included a manual override into our considerations. Another approach would be to take the Digital Twin only as expert system and let the building operator be the sole responsible for decision-making; this approach also seems the safest from a legal standpoint.

\section{CONCLUSIONS}

This paper has put forward a practical example of a Digital Twin controlling the people flow from and to a preserved heritage building. This was realized by means of an Agent-Based Simulation on top of a Building Information Model, which receives data from physical sensors installed around the building (people inflow) and changes the state of physical actuators (retractable barriers controlling outflow). The reverse way - namely interfacing physical controls (buttons, switches) to this virtual representation is also possible.

After analyzing the State of the Art, the authors conclude that the few Agent Based Simulation currently implemented in heritage buildings do not take into account this physical interface with the building. A second aspect which is yet to be covered is the introduction of a non-planned use, e.g. via physical override or insertion of new rules for the computa- 
tion of state actuation at runtime; in the latter we see a field for future research still to come.

\section{REFERENCES}

Brandi, C and Basile, G 2005, Theory of Restoration, Istituto centrale per il restauro (ISBN 9788840440897), Rome (Italy)

Helbing, D, Farkas, I and Vicsek, T 2000, 'Simulating dynamical features of escape panic', Nature, 407, p. 487-490

Hu, H, Luo, Z, Chen, Y, Bian, Q and Tong, Z 2017 'Integration of Space Syntax into Agent-Based Pedestrian Simulation in Urban Open Space', Proceedings of CAADRIA 2017, Suzhou (China), pp. 325-334

Al Qattan, E, Galanter, P and Yan, W 2016 'Developing a Tangible User Interface for Parametric and BIM Applications Using Physical Computing Systems', Proceedings of eCAADe 2016, Oulu (Finland), pp. 621630

Sharma, SB and Tabak, V 2008 'Rapid Agent Based Simulation of People Flow for Design of Spaces Analysis', Proceedings of DDSS 2008, Eindhoven (Netherlands)

Simeone, D, Coraglia, UM, Cursi, S and Fioravanti, A 2016 'Behavioural Simulation for Built Heritage Use Planning', Proceedings of eCAADe 2016, Oulu (Finland), p. 503-510

Trento, A, Fioravanti, A and Rossini, F 2014 'Health and Safety Design by means of a Systemic Approach', Proceedings of eCAADe 2014 (Volume 1), Newcastle, pp. 633-642 